\title{
Petits et grands producteurs de lait dans les Andes équatoriennes: une comparaison des performances économiques
}

\author{
Alvaro Salazar ${ }^{1}$ et Hubert Cochet $^{2, *}$ \\ ${ }^{1}$ Consultant, Quito, Équateur \\ ${ }^{2}$ AgroParisTech, UFR agriculture comparée, UMR Prodig, Paris, France
}

\begin{abstract}
Résumé - Dans les Andes équatoriennes, les cantons de Montúfar et Huaca ont connu une spécialisation laitière assez poussée et qui a concerné à la fois les grandes exploitations issues des haciendas «historiques » et un grand nombre de petites et moyennes exploitations en partie issues des processus de réforme agraire. Après avoir reconstitué les modalités de cette spécialisation à deux vitesses et explicité l'état de la différenciation actuelle des systèmes de production, cet article propose une comparaison des performances techniques et économiques des principaux types d'exploitations engagées dans la spécialisation laitière. On démontre alors que, en s'emparant de certains des moyens de production et techniques récentes utilisées depuis plus longtemps dans les grandes exploitations spécialisées, les petites et moyennes exploitations ont mis en œuvre des processus de production laitière plus intensifs en travail et hautement productifs.
\end{abstract}

Mots clés : système agraire / valeur ajoutée / productivité / lait / Équateur

\begin{abstract}
Small and large-scale milk producers in the Ecuadorian Andes: a comparison of economic performance. In the Ecuadorian Andes, the Montúfar and Huaca Cantons have specialised in dairy production. This specialisation concerns large farms, which originated from historic haciendas, and many small and medium-sized farms, which developed partly because of the agrarian reform. After discussing the reasons for this two-tier specialisation and explaining the current differentiation of production systems, this article compares the technical and economic performances of the main types of farms specialising in milk production. We show that, by adopting certain means of production and new techniques implemented by large historic dairy farms, small and medium-sized farms have developed milk production processes that are both labour-intensive and highly productive
\end{abstract}

Keywords: agrarian system / value added / productivity / milk / Ecuador

\section{Introduction}

Les propriétaires d'haciendas (ce terme désignant, en Équateur, les unités de production de grande taille dont l'origine est l'hacienda traditionnelle «historique » établie pendant la période coloniale) et les agriculteurs-entrepreneurs (à la tête d'exploitations également de grande taille, fonctionnant sur le modèle de la firme, mais de constitution récente) représentent en Équateur $15,5 \%$ des unités de production. De son côté, l'agriculture familiale représente $84,5 \%$ des exploitations (INEC, 2000). Les premiers concentrent $80 \%$ des terres et $63 \%$ de l'eau, alors que la seconde n'accède qu'à $20 \%$ de la terre et $37 \%$ de l'eau d'irrigation (Jara, 2009). Dans les Andes de l'Équateur, les grandes exploitations de type «hacienda», souvent tournées vers la production de lait, occupent encore aujourd'hui une bonne partie des meilleures terres, lovées au

\footnotetext{
$\bar{*}$ Auteur de correspondance $:$ hubert. cochet@agroparistech.fr
}

fond du sillon inter-andin qui sépare les cordillères orientale et occidentale. Il s'agit surtout d'exploitations de nature capitaliste, étant donné que, d'une part, l'objectif principal est de maximiser le taux de rentabilité du capital investi et que, d'autre part, le fonctionnement de l'exploitation repose surtout sur l'emploi de travailleurs salariés.

Mais en marge de ces haciendas, sur les versants plus escarpés, on trouve également de nombreuses exploitations familiales petites et moyennes. Bon nombre de ces agriculteurs pratiquent également l'élevage bovin laitier, mais dans le cadre de systèmes de production beaucoup plus intensifs en travail. Leur production est pourtant écoulée sur le même marché que celle des grands hacendados. Et l'apport des petites et moyennes exploitations à la production laitière nationale rivalise désormais avec celui des haciendas.

C'est sur le constat de cette dichotomie qui caractérise souvent les bassins laitiers des Andes équatoriennes que repose cet article. Le fait que des unités de production de taille très 
différente se soient engagées dans la même spécialisation laitière - on verra pourquoi - fournit en effet une occasion de choix pour s'interroger sur les performances comparées de ces différents types d'exploitations. Les plus grandes, celles dont le fonctionnement repose surtout sur la force de travail salariée sont-elles les plus efficaces, et de quels points de vue? Les exploitations agricoles de plus petite taille, au contraire, et reposant surtout sur la force de travail familiale, sont-elles en mesure d'être également performantes, et selon quels critères? L'occasion nous sera donc donnée de contribuer, sur la base d'un cas concret, au débat, toujours vif, sur ce sujet (Binswanger, 1995 ; Cochet, 2015 ; Rosset, 1999).

Les cantons de Montúfar et Huaca, situés dans la partie andine de la province de Carchi, constitueront notre terrain d'étude (Fig. 1). Il s'agit d'un petit bassin laitier particulièrement intéressant car il illustre bien la diversité agronomique, économique et sociale de la production laitière équatorienne. De même, il fournit aussi un exemple marquant de l'écart qui sépare l'agriculture familiale de l'agriculture de firme, notamment dans le cas du secteur laitier, écart très marqué tant au niveau de la taille des exploitations (de 1 à 500 hectares) que de la production de lait par exploitation (de 10 à 1500 litres de lait par jour). Cette région présente aussi une remarquable unité historique, à la fois en termes d'évolution du mode d'exploitation du milieu, en matière de différenciation interne des systèmes de production et d'évolution des rapports sociaux, une entité territoriale qui se laisse bien appréhender, comme nous le verrons, en terme de système agraire (Cochet, 2011). C'est dans ces deux cantons que les enquêtes approfondies qui servent de base à cet article ont été réalisées. Il s'agit de 254 études de cas approfondies qui y ont été réalisées entre novembre 2009 et février 2011, dans le cadre d'une recherche doctorale conduite par Salazar sous la direction de $\mathrm{H}$. Cochet (Salazar, 2016).

Après avoir présenté sommairement le zonage agroécologique de ce bassin laitier (première partie), zonage qui sous-tend, nous le verrons, la structuration sociale de la région, nous retracerons dans une seconde partie les modalités historiques de la spécialisation laitière à deux vitesses qu'elle a connue. La troisième partie fera le point sur l'état actuel de la différenciation en s'appuyant sur une typologie des exploitations agricoles, reflétant à la fois leurs trajectoires historiques et les modalités de leur fonctionnement actuel. Dans la quatrième partie, nous nous appuierons sur cette typologie pour comparer les performances économiques des différents types d'exploitations.

\section{Zonage agro-écologique des cantons de Montúfar et Huaca}

La région étudiée (Fig. 2) qui correspond aux limites des cantons de Montúfar et Huaca, consiste en un vaste bassin (d'altitude) situé entre les deux cordillères orientale (páramos de Guaranda) et occidentale (páramos del Ángel): le haut bassin du Rio Minas et de ses affluents, bien arrosé et tapissé d'une mosaïque de prairies temporaires et de parcelles de culture (surtout de la pomme de terre en altitude, cultures plus diversifiées un peu plus bas). La densité des haies confère aux versants de ce bassin un caractère de bocage à mailles denses, tandis que le centre du bassin, situé à plus basse altitude $(2800 \mathrm{~m})$ et largement dominé par la grande propriété, est caractérisé par un parcellaire de plus grande taille et une moindre présence des haies.

\subsection{La dépression centrale, dominée par les haciendas}

Le fond de ce bassin d'altitude - autour de 2700-2800 mest caractérisé par un relief faiblement marqué avec des pentes qui dépassent rarement $20 \%$. Les grandes propriétés ou haciendas - plusieurs centaines d'hectares- sont localisées sur ces espaces. On y observe des parcelles de grande taille, séparées les unes des autres par des barbelés ou parfois des haies vives, vastes prairies temporaires dans lesquelles pâturent des troupeaux bovins de 20 à 60 têtes (Fig. 3). Certaines prairies disposent d'un système d'irrigation sous pression, surtout pour luter contre le gel, et d'abreuvoirs mobiles pour les animaux. Le renouvellement des prairies et leur entretien sont réalisés au tracteur.

\subsection{Sur les versants des cordillères, l'agriculture familiale}

À partir de la dépression centrale, aussi bien en direction de la cordillère orientale que de la cordillère occidentale, nous retrouvons un relief beaucoup plus accentué, situé entre 2800 et $3000 \mathrm{~m}$ d'altitude, et où les parcelles sont de plus petite taille, encloses, et donnant lieu à un paysage de bocage (Fig. 3). $\mathrm{Au}$ côté des prairies temporaires, qui occupent une bonne moitié de la surface, on observe de nombreuses cultures, au premier rang desquelles la pomme de terre, cette culture faisant office, comme nous le verrons, de précédent cultural pour l'implantation d'une nouvelle prairie temporaire. Comme dans le fond de la vallée, la conduite des animaux repose en grande partie sur l'utilisation des clôtures électriques, même si l'on peut observer des petits troupeaux conduits au piquet. Les troupeaux sont de plus petite taille avec une quinzaine d'animaux tout au plus, pâturant sur une même parcelle.

\subsection{La frontière agricole d'altitude et les nouveaux espaces pour l'installation des paysans}

L'étage situé plus haut, entre 3000 et 3400 m d'altitude, est plus accidenté encore, et recèle encore des fragments de forêts primaires et secondaires (Fig. 3). Là où la pente le permettait, ces forêts ont été défrichées pour leur mise en culture, en dépit de l'interdiction des autorités. Les agriculteurs y pratiquent d'abord l'abattis-brûlis et, une fois la surface dégagée, y plantent des pommes de terre, après un passage d'araire. Un deuxième cycle de pomme de terre peut suivre avant que la parcelle ne soit convertie en prairie temporaire pendant cinq ans environ. La culture principale est ici la pomme de terre bien que les rendements y soient, du fait de l'altitude, moins élevés que dans les zones précédentes.

\section{Les deux visages de la spécialisation laitière de la Sierra}

\subsection{Les prémices de la spécialisation laitière des haciendas}

C'est au milieu du $\mathrm{XX}^{\mathrm{e}}$ siècle que la production laitière connaît sa première phase de développement important dans les haciendas de la région. Auparavant, c'est surtout autour de 


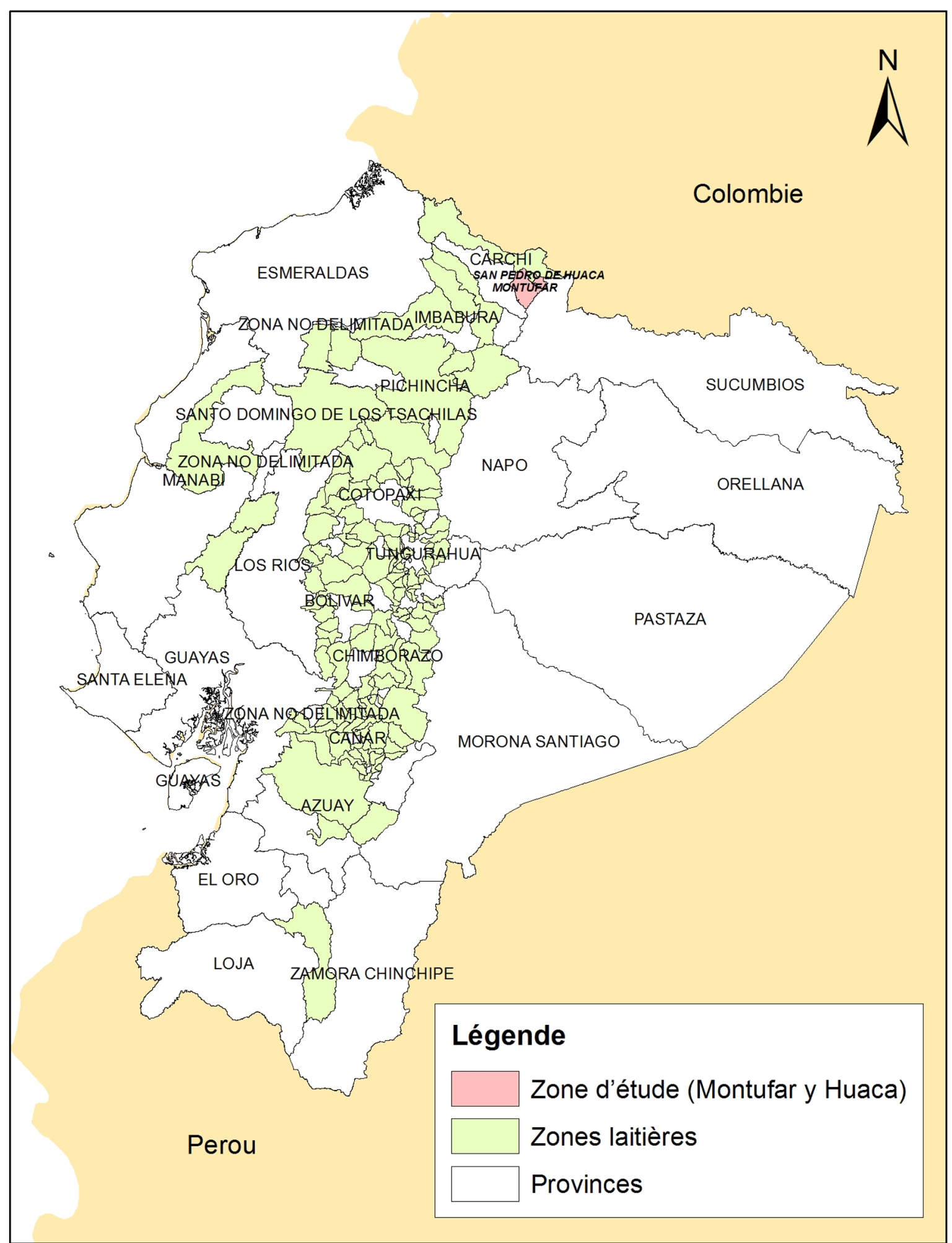

Fig. 1. Les cantons de Montúfar et Huaca, Province de Carchi, dans la géographie laitière équatorienne. Source: Salazar (2016). Fig. 1. Montúfar and Huaca Cantons, Carchi Province, in the dairy belt of Ecuador. 


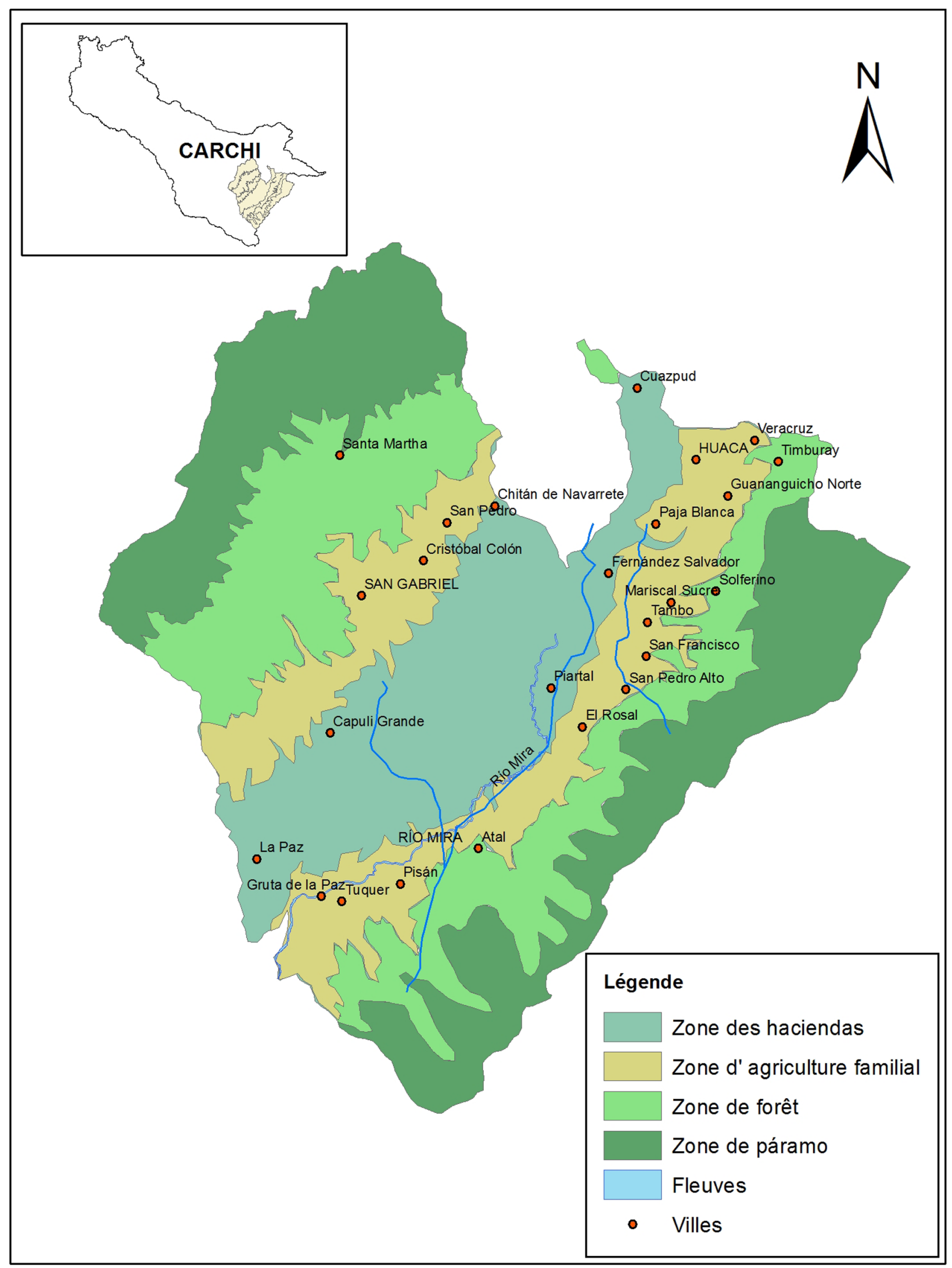

Fig. 2. La région étudiée: les cantons Montúfar et Huaca, Province du Carchi, Équateur. Source : Salazar (2016).

Fig. 2. Study sites: Montúfar and Huaca Cantons, Carchi Province, Ecuador. 

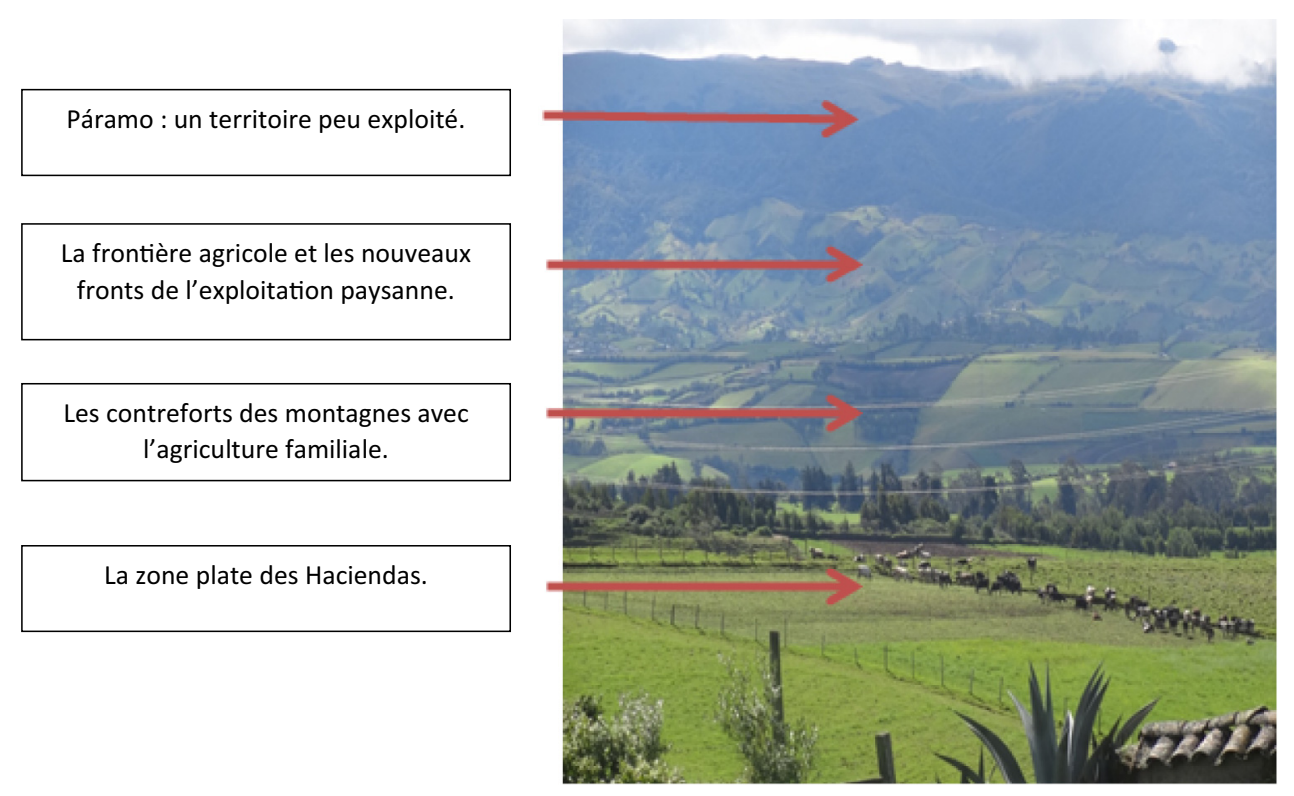

Fig. 3. Étages écologiques et paysage dans les cantons de Montúfar et Huaca. Source: auteurs.

Fig. 3. Ecological succession and landscape in the Montúfar and Huaca Cantons.

la céréaliculture que ces grands domaines plaçaient leur production sur le marché, notamment la production de blé pour la fabrication de farine, et d'orge pour les brasseries colombiennes. Mais l'installation de la brasserie nationale équatorienne, les pressions exercées par les industriels pour obtenir l'importation à bas prix de matière première, ainsi que les politiques colombiennes de soutien à la production nationale dissuadèrent les propriétaires des haciendas du Carchi de continuer à produire des céréales. À la même époque, de nouvelles opportunités de marché sont développées dans les villes proches de Quito et Ibarra, qui constituaient déjà des marchés très importants pour les fromages, le beurre et la crème produits dans les haciendas.

Dès le début des années 1940, les propriétaires vont ainsi commencer à introduire de nouvelles races bovines afin d'accroître la production (Barsky, 1983). La production fourragère progresse, notamment grâce à l'installation de prairies temporaires à base de ray grass, prairies précédées, lors de leur renouvellement, par un cycle de pomme de terre.

Sur les versants dominant le fond de la vallée, de très nombreuses exploitations de très petite taille gravitaient autour des haciendas. Afin d'éviter le départ de la force de travail de la Sierra vers les plantations de cacaoyers situées sur la côte à la fin du XIX ${ }^{\mathrm{e}}$ siècle, puis, plus tard, vers les plantations de bananiers, les propriétaires des haciendas du Carchi avaient en effet retenu leurs journaliers en multipliant le nombre de huasipungos. Ces derniers correspondaient à de petits lopins de terre appartenant à l'hacienda dont les bénéficiaires, les huasipungueros - d'anciens journaliers - pouvaient disposer les weekends en plus de leur maigre salaire obtenu de leur travail en semaine pour l'hacienda. Cela allait provoquer la généralisation de la petite tenure paysanne autour des haciendas.

Les huasipungueros et les paysans qui avaient accédé à la terre à leur propre compte, souvent associés en coopératives, produisaient surtout des cultures vivrières, notamment la pomme de terre. Ils commencèrent pourtant à pratiquer eux- aussi un élevage bovin, non seulement pour produire du lait pour la consommation familiale mais aussi pour fertiliser les parcelles cultivées et se constituer progressivement un petit capital.

\subsection{La réforme agraire et ses conséquences}

Voyant leurs exploitations amputées d'une partie de leur surface au cours des processus de réforme agraire des années soixante et soixante-dix, mais conservant toujours les meilleures terres du fond de vallée, les grands propriétaires s'adaptent en réduisant la taille de leurs exploitations et en s'engageant plus avant dans la production laitière.

Sur une surface de quelques dizaines ou centaines d'hectares particulièrement bien situés - relief peu accidenté propice à la motorisation, densité du réseau de voies de communication, ressources en eau abondantes - la spécialisation laitière était sans doute l'option la plus évidente et la plus rentable à mettre en œuvre. Elle permettait de conserver, sur des surfaces relativement modestes au regard de l'hacienda coloniale, une position largement dominante, du point de vue à la fois économique, social et politique, au regard des exploitations de beaucoup plus petite taille des environs. Par ailleurs, les pertes foncières des haciendas concernent surtout leur périphérie montagneuse, c'est-à-dire des terres anciennement cultivées en vivrier par les travailleurs de l'hacienda. C'est ainsi que le «cour» de l'hacienda, lieu de prédilection de l'élevage laitier, devient une unité de production beaucoup plus spécialisée dans le lait que ne l'était, toutes choses égales par ailleurs, l'ancien grand domaine dans sa configuration pré-réforme agraire. La réforme agraire se traduit ainsi par une spécialisation laitière de facto de ce qui reste des anciennes haciendas, les terrains les moins favorables de l'hacienda, support d'une grande partie de la production vivrière, étant désormais aux mains des bénéficiaires de la réforme agraire. 
En permettant à un nombre croissant d'anciens huasipungueros de devenir maître chez eux, la réforme agraire ouvre la voie à un timide processus d'accumulation, pour lequel l'élevage laitier sera, lui aussi, appelé à jouer son rôle...

\subsection{Les haciendas investissent dans de nouvelles techniques plus productives et accentuent leur spécialisation laitière}

À la fin des années 1970 et au début des années 1980, les haciendas du Carchi, déjà tournées vers l'élevage laitier, entrent dans un processus de spécialisation caractérisé par des innovations techniques importantes. Tandis que la motomécanisation se généralise y compris pour la traite, les veaux sont séparés précocement de leur mère et nourris, en partie, avec de la poudre de lait. Les propriétaires des haciendas s'entourent de personnels spécialisés (70\% comptent un vétérinaire dans leurs rangs) et le recours à des rations équilibrées pour chaque lot se développe. C'est à cette époque que les premières inséminations artificielles avec des semences importées sont effectuées. Ainsi, dans les haciendas laitières du Carchi, les rendements moyens laitiers progressent de 4 litres par jour et par vache en 1962 à 13 litres en 1982 (SICA/ INEC/MAGAP, 2002) puis 15 litres en 1990 (INEC, 2000).

Concernant la conduite des pâturages, à partir des années 1970, certaines haciendas vont mettre en place des systèmes d'irrigation par aspersion, notamment dans les prairies dominées par le kikuyo (Pennisetum clandestinum), situées dans la dépression centrale où les gelées sont fréquentes. Clôtures en fil de fer barbelé et clôtures électriques se généralisent, facilitant ainsi la conduite des animaux.

\subsection{Le développement de la production paysanne de lait, dans le sillage des haciendas}

À la fin des années 1970, l'activité principale des petites unités de production paysannes des Andes du Carchi n'était pas l'élevage bovin laitier et les petits volumes de lait produits étaient surtout destinés à l'autoconsommation sous forme de lait cru ou de fromages artisanaux (cuajadas et quesillos). Toutefois, la production de lait par la paysannerie du Carchi connaît un certain élan à partir du début des années 1980 lorsque de nombreux agriculteurs commencent à intégrer des circuits courts de commercialisation, notamment celui des fromages frais écoulés sur les marchés (Pérez, 2002 ; Salazar, 2016)

Les agriculteurs commencent en effet à mettre en œuvre certaines des pratiques utilisées avec succès dans les haciendas, dont le semis de mélanges fourragers, l'utilisation de barbelés et le recours à d'autres pratiques associées à la conduite des animaux. Alors que jusque dans les années 1980, la plupart continuaient à élever les veaux (mâle comme femelle) avec leur mère pendant les 3 à 6 premiers mois, à partir des années 1990, les paysans s'engagent dans le sens d'une spécialisation plus poussée : séparation des veaux de leur mère quelques jours après la naissance et vente précoce des veaux mâles pour faire davantage de place aux vaches laitières.

Le développement progressif de la production laitière dans les exploitations de petite taille, développement qui ne conduit que rarement - ou jamais - à une spécialisation complète (on renouvelle les prairies temporaires avec un cycle de pomme de terre) semble se réaliser dans le sillage de la spécialisation laitière des grandes exploitations, et avec un temps de retard. Il ne s'agit pourtant pas d'une simple diffusion, par imitation, de l'innovation. Certains moyens de production déjà accessibles aux haciendas et certaines pratiques ont retenu l'attention des agriculteurs des environs, d'autre, non ( $c f$. infra). Le résultat en est la mise au point de façons de faire parfois plus efficaces que celles mises en place dans les grandes exploitations (par exemple le pâturage en double-fil), notamment en termes de création de richesse par unité de surface ( $c f$. infra).

Enfin, dès lors que le prix du lait restait attractif, les exploitations paysannes des environs n'avaient-elle pas intérêt, elles aussi, à développer autant que faire se peut la production laitière? Certes elles ne bénéficiaient pas des conditions favorables des grandes exploitations du fond de vallée, mais la production laitière était en mesure de valoriser, jour après jour, 365 jour sur 365 , une force de travail familiale abondante et dont le coût d'opportunité restait très faible, du fait de la rareté des opportunités de travail rémunéré et stable à l'extérieur. Un tel développement de la production laitière dans les petites exploitations a d'ailleurs été décrit dans d'autres régions des Andes équatoriennes (Deslandes et Micoulaud, 2006 ; Huttel et al., 1999).

\subsection{Une protection croissante du secteur laitier}

Un facteur déterminant de la spécialisation laitière a été, sans aucun doute, le niveau de protection du marché intérieur. En 1995, en pleine période de libéralisation des marchés, le gouvernement établit un système d'intervention pour stabiliser les prix de certains produits stratégiques, de manière à ce que la réduction des droits de douane sur les produits importés n'entraîne pas la chute du prix de certains produits nationaux comme le lait. Le lobbying de la corporation des éleveurs - en fait, les propriétaires des grandes haciendas d'élevage laitiera alors joué un rôle de premier plan dans la mise en œuvre de ces politiques destinées à protéger le secteur et à restreindre les importations de lait et de produits laitiers (Estrada et Paladines, 2006). Entre 2002 et 2007, ces mesures ont permis de fixer un prix du lait de 0,25 dollar par litre. Début 2008, ce prix augmenta jusqu'à 0,28 dollar avant d'atteindre un prix minimum garanti de 0,35 dollar par litre (MAGAP, 2012). Les éleveurs obtiennent même en 2010, un prix minimum de 0,39 dollar par litre auquel s'ajoutent des compléments liés aux normes sanitaires et de qualité qui élèvent le prix final à 0,45 dollar, un prix largement supérieur au prix mondial.

Ce sont bien sûr les propriétaires d'haciendas qui parviennent le mieux à bénéficier de ces prix élevés compte tenu des grands volumes commercialisés et de leur accès direct aux industries laitières. Mais il est certain que le lobby puissant des plus grands éleveurs laitiers a aussi servi la cause de cette économie paysanne laitière en formation. Certes, les petits producteurs doivent le plus souvent écouler leur production en passant par des intermédiaires prélevant leur part au passage, mais, dans tous les cas, les paysans bénéficient aussi des prix garantis et de la stabilité qu'ils leur procurent. Par ailleurs, de nouvelles formes d'organisation des communautés se mettent en place pour améliorer l'accès aux marchés en installant des centres de collecte et de refroidissement du lait. 


\section{Diversité actuelle et principales caractéristiques des exploitations agricoles des cantons de Montúfar et Huaca}

\subsection{Trajectoires historiques et différenciation: une typologie des exploitations agricoles}

Le parcours historique qui précède décrit les processus de structuration agraire et les changements survenus tant en termes d'accès à la terre et aux moyens de production qu'en termes de pratiques d'élevage. Les différentes catégories socioéconomiques qui caractérisent les cantons de Montúfar et Huaca aujourd'hui sont le produit de cette histoire. Les résultats présentés ci-dessous reposent sur 254 entretiens approfondis réalisés auprès de différents types d'agriculteurs, préalablement identifiés grâce à cette approche historique de la région (supra). Le choix des exploitations étudiées a été raisonné de façon à rendre compte de cette diversité de situation. On peut alors classer et ordonner cette diversité en huit situations assez contrastées. Il s'agit essentiellement :

- des haciendas « intensives » (type 1, 7 cas étudiés). Elles résultent des processus de division des grandes haciendas. Leurs propriétaires ont, pour la plupart, acheté ces grandes exploitations au moment de leur division. Ils mettent en valeur ces terres en pratiquant un élevage bovin laitier intensif (mélanges fourragers à haut rendement, irrigation sous pression, complémentation, amélioration génétique, équipement de traite performant, emploi de logiciels comptables, etc.). Pour ces grands éleveurs, la culture de la pomme de terre est surtout considérée comme un moyen de renouveler les pâturages. Elle est d'ailleurs sous-traitée moyennant des contrats à part de fruit passés avec des producteurs spécialisés (type 4 ci-dessous);

- des Haciendas «extensives» (type 2, 3 cas étudiés). Il s'agit généralement des grandes propriétés qui appartiennent aux héritiers des plus anciennes haciendas de la région. De plus grande taille que les haciendas du premier groupe (200 à $500 \mathrm{ha}$ ) mais moins nombreuses, et suivant des dynamiques de production plus anciennes, leurs propriétaires ont très peu investi dans la modernisation de leur outil de production. L'élevage pratiqué est plus extensif; quelques parcelles sont encore dédiées aux céréales, celles en pommes de terres cédées en location le temps de renouveler les prairies;

- des exploitations moyennes récemment constituées (type 3, 14 cas étudiés). Ce sont des exploitations patronales de 30 à 80 hectares dont l'origine n'est pas le patrimoine hérité, mais le résultat de processus de capitalisation récents dans d'autres secteurs de l'économie. Ces producteurs cherchent également à accroître la production laitière moyennant la mise en place de techniques comparables à celle des haciendas intensives du type 1 (amélioration génétique, accroissement des rendements des prairies, irrigation, salle de traite, etc.), bien que la diversification de la production soit aussi recherchée;

- des entrepreneurs producteurs de pomme de terre (type 4, 6 cas étudiés). Il s'agit d'un petit groupe de producteurs de pommes de terre, locataires de terres, qui écoulent leur production auprès des industries productrices de chips avec lesquelles ils ont signé des contrats de commercialisation. Ces producteurs disposent de capitaux conséquents et cultivent des surfaces importantes en pomme de terre. Pour ce faire, ils prennent en location les parcelles que les haciendas leur confient pour renouveler leur prairies temporaires et les font travailler par des équipes de journaliers ;

- des paysans ex-huasipungueros âgés qui ont capitalisé (type 5,62 cas étudiés). Nombre d'entre eux, bénéficiaires de la réforme agraire, ont pu acheter de surcroît des terres, de sorte qu'ils se trouvent aujourd'hui à la tête d'exploitations de 10 à 30 hectares et possèdent des troupeaux bovins conséquents (20 à 80 têtes). Eux aussi sont engagés dans un processus d'intensification laitière (fourrage amélioré, insémination artificielle, sevrage précoce, complémentation) mais leur niveau d'équipement est moindre et l'intensification repose aussi sur une forte sollicitation de la force de travail familiale, notamment pour la traite qui reste généralement manuelle. Ils cultivent également de la pomme de terre à la fois pour renouveler les prairies, assurer l'autoconsommation familiale, engraisser quelques cochons et vendre le surplus sur le marché. Ce groupe comprend également les paysans qui ont accédé à la terre en formant des coopératives sans jamais avoir de rapports directs avec les haciendas;

- des paysans héritiers (type 6,93 cas étudiés). Ils sont pour la plupart les fils des agriculteurs du groupe précédent, qui ont déjà reçu leur héritage et se sont installés avec leurs familles : la surface dont ils disposent étant plus faible que celle du groupe précédent, ces paysans peinent à assurer les besoins de leur famille et doivent aussi vendre leur force de travail à l'extérieur. Ils misent cependant sur la production laitière en y consacrant beaucoup de travail, à défaut de pouvoir y investir du capital. L'intensification de l'élevage repose alors, par exemple, sur un pâturage «séré» où les quelques vaches à la pâture sont conduites au fil: un fil électrique devant, déplacé régulièrement, et un fil électrique derrière pour éviter surpâturage et piétinement. Certains cultivent la pomme de terre sur les parcelles d'autrui - parfois celles de leurs parents - moyennant des contrats à part de fruit ;

- des paysans partidarios ou «métayers» (type 7, 45 cas étudiés), fils de bénéficiaires de la réforme agraire. Ils n'ont pas encore reçu leur héritage et cultivent la pomme de terre sur les parcelles d'autres exploitants - souvent leur propre père - moyennant des contrats à part de fruit. Ceux qui parviennent à capitaliser élargissent les surfaces cultivées à part de fruit sur les parcelles des exploitations voisines;

- des paysans journaliers (type 8, 24 cas étudiés). Beaucoup sont des enfants d'anciens huasipungueros, qui n'ont pas encore reçu de terres en héritage et ne disposent pas du capital circulant minimum pour cultiver la pomme de terre à part de fruit ou en métayage. Appartiennent aussi à cette catégorie les agriculteurs âgés qui n'ont jamais eu accès à la terre ou des agriculteurs paupérisés qui ont vendu leurs terres.

\subsection{Accès à la terre et assolement-type des différents systèmes de production}

Malgré les épisodes de réforme agraire des années 1960 et 1970 , la concentration de la terre est patente, comme l'illustre le Tableau 1. 
Tableau 1. Surface et assolement type par système de production.

Table 1. Cropped area and cropping pattern according to the different cropping systems.

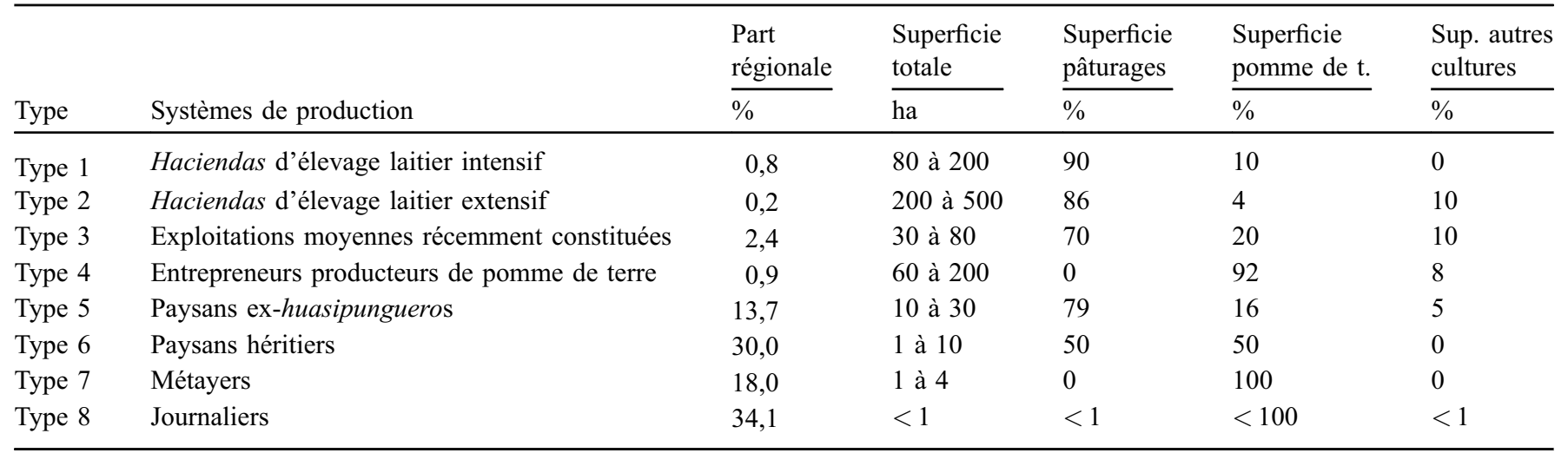

Pour chaque type d'exploitation, une gamme de surface a été indiquée, correspondant à la dispersion observée au sein de chaque type. La répartition (en \%) de l'assolement entre surfaces en pâturages, pomme de terre et autres cultures est assez homogène au sein de chaque type ; les chiffres indiqués correspondant aux moyennes calculées pour chaque type (254 études de cas réalisés entre novembre2009 et février 2011). (Source: auteurs)

Tableau 2. La force de travail dans les différents systèmes de production identifiés

Table 2. Work force in the different production systems

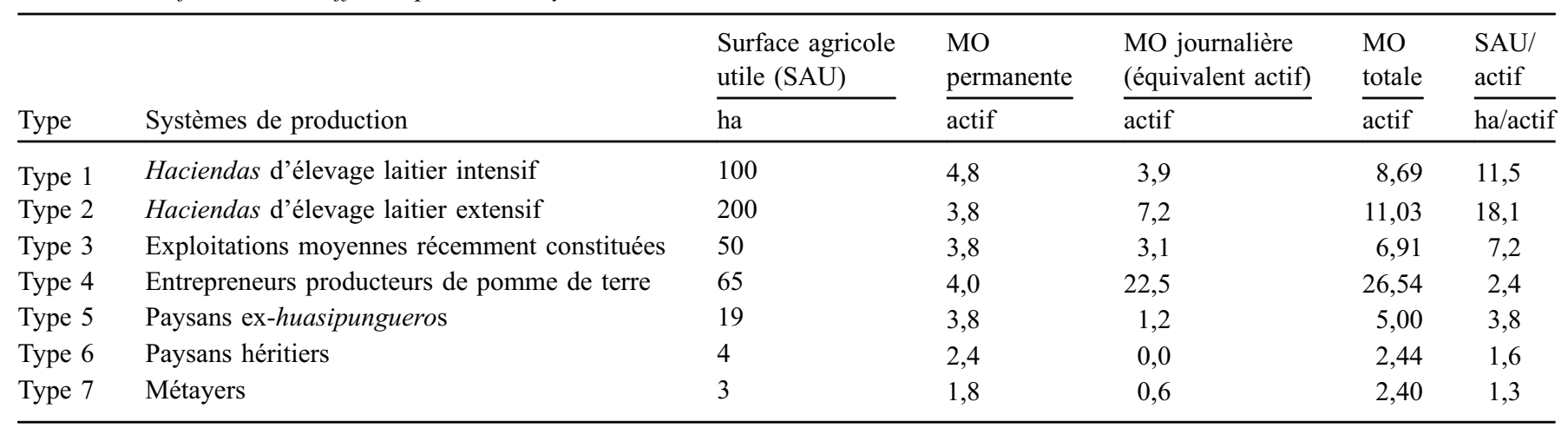

Les chiffres indiqués pour chaque type correspondent ici à un archétype construit (modélisation) pour une surface donnée, par exemple pour une exploitation de 100 ha dans le cas des haciendas d'élevage laitier intensif (type 1) (études de cas réalisés entre novembre 2009 et février 2011). (Source : auteurs)

Ce tableau montre que plus la surface disponible est grande, plus vaste est l'étendue des pâturages installés sur l'ensemble de l'exploitation. Les grands propriétaires consacrent donc une plus grande surface à la production de prairies pour le bétail bovin laitier, puisque c'est l'activité qu'ils privilégient. Dans les exploitations paysannes, en revanche, et malgré l'intérêt grandissant pour la production laitière, prédomine la logique de la sécurité alimentaire et de la limitation de risques. On y cultive encore beaucoup de pomme de terre.

\subsection{Le travail}

S'agissant de la force de travail employée dans chaque type d'exploitation, le Tableau 2 montre que dans les haciendas d'élevage laitier extensif, une unité de travail peut gérer 18 hectares, tandis que dans les haciendas d'élevage laitier intensif, cette surface se réduit à 7 ha par actif. Dans les exploitations de grande taille (type 1, type 2), quand on ne prend en compte que la surface destinée aux pâtures et donc à la production de lait - sans considérer les surfaces destinées à la pomme de terre - les besoins en travail se réduisent à 3 actifs pour 100 hectares. Cela est dû en grande partie à la motorisation, tant pour les tâches de traite que pour les tâches d'entretien des parcelles. Pour les petits agriculteurs propriétaires, dont les parcelles sont situées sur les parties les plus escarpées, la traite se fait manuellement et la gestion du bétail et des prairies requiert un travail plus constant et régulier.

La pomme de terre, quant à elle, est presque toujours cultivée en contrat à part de fruit dans les grandes exploitations, tandis que dans les exploitations familiales, les tâches sont surtout effectuées par la main-d'œuvre familiale, secondée parfois par le recours à des journaliers pour les pics de travail liés à la culture de la pomme de terre.

\section{Une comparaison des résultats économiques}

Seul un petit nombre d'indicateurs a été retenu dans le cadre de cette comparaison, parmi lesquels le rendement laitier 
Tableau 3. Rendement du lait selon les différents systèmes de production identifiés

Table 3. Milk production according to the different cropping systems.

\begin{tabular}{|c|c|c|c|c|c|c|c|}
\hline & $\begin{array}{l}\text { Superficie de } \\
\text { pâture }\end{array}$ & $\begin{array}{l}\text { Nombre } \\
\text { d'animaux }\end{array}$ & $\begin{array}{l}\text { Densité } \\
\text { animale }\end{array}$ & $\begin{array}{l}\text { Vaches en } \\
\text { production }\end{array}$ & $\begin{array}{l}\text { Pourcentage de } \\
\text { vaches en lactation }\end{array}$ & $\begin{array}{l}\text { Rendement/ } \\
\text { vache }\end{array}$ & $\begin{array}{l}\text { Rendement/ } \\
\text { ha }\end{array}$ \\
\hline Systèmes de production & hectare & têtes $^{1}$ & tête/ha & $\overline{\text { tête }}$ & $\overline{\%}$ & $\overline{\text { litres/vache }}$ & litres/ha/an \\
\hline Haciendas d'élevage laitier extensif & 172 & 153 & 1 & 70 & 46 & 11 & 1693 \\
\hline Exploitations moyennes récemment constituées & 35 & 66 & 2 & 38 & 58 & 16 & 6164 \\
\hline Paysans exhuasipungueros & 15 & 42 & 2,8 & 22 & 53 & 14 & 7227 \\
\hline
\end{tabular}

Les chiffres indiqués dans ce tableau correspondent aux archétypes correspondant aux types 1,2,3,5 et 6 , engagés dans la production laitière et déjà présentés dans le Tableau 2.

${ }^{1}$ Vaches adultes

(Source : auteurs)

Tableau 4. Valeur ajoutée nette et productivité des facteurs dans les différents systèmes de production identifiés.

Table 4. Added value and factors productivity according to the different cropping systems.

\begin{tabular}{|c|c|c|c|c|c|c|c|}
\hline & & $\begin{array}{l}\text { Surface } \\
\text { utile } \\
\end{array}$ & $\begin{array}{l}\text { Main-d'œuvre } \\
\text { agricole } \\
\end{array}$ & $\begin{array}{l}\text { VAN/ } \\
\text { an } \\
\end{array}$ & $\begin{array}{l}\text { VAN/ } \\
\text { ha } \\
\end{array}$ & $\begin{array}{l}\text { VAN/ } \\
\text { actif } \\
\end{array}$ & $\begin{array}{l}\text { VAN/ } \\
\text { jour } \\
\end{array}$ \\
\hline Type & Systèmes de production & ha & Actif & dollars* & $\overline{\text { dollars }}$ & $\overline{\text { dollars }}$ & dollars \\
\hline Type 1 & Haciendas d'élevage laitier intensif & 100 & 8,7 & 189500 & 1900 & 21800 & 84 \\
\hline Type 2 & Haciendas d'élevage laitier extensif & 200 & 11,0 & 150400 & 800 & 13600 & 52 \\
\hline Type 3 & Exploitations moyennes récemment constituées & 50 & 6,9 & 94100 & 1900 & 13600 & 52 \\
\hline Type 6 & Paysans héritiers & 4 & 2,4 & 10500 & 2600 & 4300 & 16 \\
\hline Type 7 & Métayers & 3 & 2,4 & 12400 & 4100 & 5200 & 20 \\
\hline
\end{tabular}

* tous les chiffres sont exprimés en dollars des États-Unis (USD) monnaie adoptée en 2000 par l’Équateur.

(par vache et par ha), la valeur ajoutée, la productivité du travail et la valeur ajoutée dégagée par ha (ou productivité de la terre). Pour plus de clarté et afin de favoriser la comparaison, les résultats techniques et économiques des systèmes de production (Tab. 3 et 4 ) sont présentés pour des archétypes - des modèles - représentant, chacun, un type d'exploitation.

Dans le Tableau 3, on peut apprécier comment la charge animale augmente au fur et à mesure que diminue la surface de pâturages disponible. Tandis qu'elle est de 0,9 vache/ha dans les haciendas d'élevage laitier extensif et de 1,9 vaches/ha dans les haciendas d'élevage laitier intensif, elle atteint 2,8 vaches/ha chez les paysans ex-huasipungeros (type 5), et même 3,3 vaches/ha dans les plus petites exploitations (type 5).

En ce qui concerne le rendement laitier à l'hectare, ce sont les paysans ex-huasipungueros, avec 7200 litres par hectare à l'année, qui sont les plus performants, suivis par les paysans héritiers avec $6200 \mathrm{l} / \mathrm{ha} / \mathrm{an}$; viennent ensuite les hacendados d'élevage intensif (de 5600 à 6200 1/ha/an) et finalement les hacendados d'élevage extensif qui ne produisent pas plus de 1700 1/ha/an. Ces résultats montrent clairement que le plus haut rendement, en dépit de leur faible surface, est obtenu par les petits producteurs. Ceux-ci parviennent en effet à produire la plus grande quantité de lait par hectare, sans pour autant acheter davantage d'alimentation complémentaire par vache, bien au contraire. Il en résulte une valeur ajoutée nette par hectare (VAN/ha) nettement supérieure dans les petites exploitations, comme en témoigne le Tableau 4.

Ces résultats proviennent d'une gestion plus intensive en travail, qui permet de tirer parti de chaque mètre carré de pâture disponible. Des pratiques telles que le double fil électrique, devant et derrière le bétail, sont essentielles, tout comme l'est la détection à temps des chaleurs, grâce à l'accompagnement au quotidien des bêtes.

Le Tableau 4 met en évidence les écarts concernant la productivité de la terre (VAN/ha) et la productivité du travail (VAN/jour) entre les différents types de producteurs, indicateurs particulièrement intéressants pour comparer les modèles agricoles (Cochet, 2015).

On voit que les producteurs qui créent le plus de richesse par unité de surface sont ceux qui produisent du lait sur des surfaces modestes (type 5 et 6) ainsi bien sûr que les producteurs de pommes de terre (type 4 et 7 ). Les entrepreneurs à la tête des plus grandes exploitations (types 1,2 et 3) dégagent une valeur ajoutée par hectare nettement moins élevée, inférieure à 2000 USD/ha.

S'agissant de la productivité du travail (VAN/actif), les résultats diffèrent bien sûr de ceux concernant la VAN/ha. La plus grande productivité du travail (mesurée par actif ou par 
journée de travail) enregistrée dans les exploitations les plus grandes est à mettre en relation avec leur niveau d'équipement permettant de gérer davantage de vaches (et d'hectares) par actif. Une hacienda intensive de type 1 peut ainsi produire plus de 20000 dollars de valeur ajoutée nette par actif par an, tandis que la productivité du travail sera deux fois moindre dans une exploitation paysanne également tournée vers l'élevage laitier (type 5 par exemple).

Étant donné que la culture de pomme de terre demande beaucoup de main-d'œuvre, les entrepreneurs-producteurs de pommes de terre (type 4), les paysans héritiers (type 6) et les métayers (type 7), génèrent moins de richesse par jour de travail par rapport aux propriétaires hacendados et paysans spécialisés dans l'élevage. Mais l'inclusion de la pomme de terre dans les systèmes de production permet de créer plus de richesse par unité de surface; en outre, parce qu'elle est créatrice d'emploi rural et agricole, elle contribue à une certaine redistribution de la richesse dans ces campagnes.

\section{Conclusion}

Le développement de la production laitière dans les Andes de la province du Carchi et l'orientation préférentielle de la majorité des exploitations agricoles en présence - grandes ou petites - dans cette production offre ainsi à l'observateur l'occasion de comparer à la fois les trajectoires et rythmes de cette progressive spécialisation et les résultats, tant techniques qu'économiques, obtenus par les différents types d'exploitations engagés dans une même spécialisation productive. Les résultats confirment encore une fois que les exploitations de petite taille valorisent en général beaucoup mieux le facteur dont elles disposent en moindre quantité, la terre, au prix d'une forte intensification en travail qui permet de relever sensiblement la valeur ajoutée produite par unité de surface. Ils montrent également que loin de se diffuser par simple imitation, les nouveaux moyens de production et nouvelles techniques dont se sont emparés les éleveurs les plus modestes ont été choisis, adaptés et réassemblés par ces derniers en systèmes de production entièrement nouveaux et très sensiblement différents, notamment en termes d'intensification en travail, de ceux dont ils étaient censé s'inspirer: les plus grandes exploitations spécialisées plus tôt dans la production laitière et ayant eu accès précocement aux nouvelles technologies ad hoc. Nul doute que le fort soutien des prix dont a bénéficié l'ensemble des éleveurs laitiers équatoriens fut un facteur décisif dans ce processus. Dès lors, une véritable convergence d'intérêts entre les haciendas laitières et les exploitations de plus petite taille a vu le jour, convergence d'intérêts autour du maintien d'un prix élevé du lait et de la protection de l'industrie laitière nationale, et non pas bien sûr autour de la question de l'accès aux ressources (terres et eau, notamment), pour lesquelles ces deux secteurs restent directement concurrents. Reste au bout du chemin la perspective attendue d'une baisse sensible du prix du lait (Salazar, 2016), baisse qui pourrait, au-delà de ces effets différenciés sur les différents types de producteurs, faire voler en éclat cette alliance « objective».

Remerciements. Les auteurs remercient la Revista de Geografía Agrícola (Mexique ; http://www.redalyc.org/revista.oa? $\mathrm{id}=757$ ) pour avoir autorisé la publication de cet article dont les figures et une partie des données figurent en espagnol dans Salazar et Cochet (2016).

\section{Références}

Barsky O. 1983. Pequeña producción y acumulación del capital. Quito: IICA, Quito.

Binswanger HP. 1995. The myth of large-farm superiority. Dissemination notes number 6. Washington DC, USA: The World Bank, Agriculture and Natural Resources Department, 5 p.

Cochet H. 2011. Origine et actualité du «système agraire » : retour sur un concept. Revue Tiers Monde 207: 97-114.

Cochet H. 2015. Controverses sur l'efficacité économique des agricultures familiales: indicateurs pour une comparaison rigoureuse avec d'autres agricultures. Revue Tiers Monde 221: 9-25.

Deslandes K, Micoulaud B. 2006. Diagnostic agraire dans la partie amont de la vallée du río Jubones- Cantons Girón et San Fernando Équateur. Rapport pour l'obtention du diplôme de l'ingénieur Agronome. INAPG. Paris-France.

Estrada R, Paladines O. 2006. El impacto de las políticas en el desarrollo lechero de los Andes. El caso de la Sierra Ecuatoriana. Quito.

Huttel C, Zebrowski C, Gondard P. 1999. Paisajes Agrarios del Ecuador. Geografía Básica del Ecuador. Tomo V, Vol 2. IPGHORSTOM- IGM- CEDIG. Quito.

INEC. 2000. Censo Nacional Agropecuario. Quito.

Jara C. 2009. Plan Nacional del Buen Vivir Rural del Ecuador 2009_ 2013. Quito (Ecuador): SEMPLADES.

MAGAP. 2012. Informe de los precios de la leche a nivel nacional 2000-2012. Quito

Pérez D. 2002. Hacia el desarrollo de territorios rurales en el norte: Análisis económico del sector lácteo del cantón Montúfar. Fondo Ecuatoriano Populorum Progressio, FEPP, Regional Ibarra.

Rosset P. 1999. The multiple functions and benefits of small farm agriculture in the context of global trade negotiations. Paulus Potterstraat 201071 DA, Amsterdam (The Netherlands): Transnational Institute, $24 \mathrm{p}$.

Salazar A. 2016. Les Andes Septentrionnales: une dynamique productive entre haciendas et paysannerie. Thèse de doctorat AgroParisTech, 338 p.

Salazar A, Cochet H. 2016. Haciendas y campesinos lecheros en el Carchi (Andes húmedos del norte del Ecuador): dinámica productiva y comparación de los resultados técnicos y económicos Revista de Geografia Agrícola http://www.redalyc.org/articulo.oa? $\mathrm{id}=75749288005$.

SICA, INEC, MAGAP. 2002. III Censo Nacional Agropecuario, resultados nacionales. Quito.

Citation de l'article : Salazar A, Cochet H. 2017. Petits et grands producteurs de lait dans les Andes équatoriennes : une comparaison des performances économiques. Cah. Agric. 26: 55004. 